\title{
The Inhibition Effects of Alkoxyl Thiourea Derivatives toward the Growth of Isolated Oscillatoria sp. from Kenyir Lake, Terengganu
}

\author{
Nor Azman Kasan ${ }^{1,2}$, Siti Zafirah Mohamad Yusof ${ }^{1}$, Naqiyyu Bazz Ramli ${ }^{3}$, Wan M. Khairul ${ }^{3}$ and Hazlina \\ Ahamad Zakeri ${ }^{3}$ \\ 1. Institute of Tropical Aquaculture (AKUATROP), University Malaysia Terengganu, Kuala Terengganu 21030, Malaysia \\ 2. School of Fisheries and Aquaculture Science, Universiti Malaysia Terengganu, Kuala Terengganu 21030, Malaysia \\ 3. School of Fundamental Science, Universiti Malaysia Terengganu, Kuala Terengganu 21030, Malaysia
}

\begin{abstract}
In controlling eutrophication phenomenon, there were conventional methods which lead to negative effects to aquatic environment. This study was aimed to investigate the usage of synthesized thiourea compounds to inhibit the growth of Oscillatoria sp. in Kenyir lake, Terengganu, Malaysia. The inhibition effects of four eco-friendly alkoxyl thiourea derivatives compounds named as $\mathrm{N}-((4-($ decyloxy$)$ phenyl)carbamothioyl)-4-methyl benzamide, $\mathrm{N}-((4-($ decyloxy$)$ phenyl)carbamothioyl)-4-nitro benzamide, 4-chloro-N-((4-(decyloxy)phenyl)carbamothioyl) benzamide and $\mathrm{N}-((4-($ decyloxy)phenyl)carbamothioyl) benzamide were examined onto the growth culture of Oscillatoria sp. These compounds were tested in $30 \mathrm{~mL}$ of Oscillatoria sp. cultures with different concentration of $16 \mu \mathrm{g} \cdot \mathrm{mL}^{-1}, 18 \mu \mathrm{g} \cdot \mathrm{mL}^{-1}, 20 \mu \mathrm{g} \cdot \mathrm{mL}^{-1}, 24 \mu \mathrm{g} \cdot \mathrm{mL}^{-1}$ and $28 \mu \mathrm{g} \cdot \mathrm{mL}^{-1}$ respectively. The treatment flasks were supplied with an aerator for 24 hours under continuous illumination at $25{ }^{\circ} \mathrm{C}$. Chlorophyll-a concentration were extracted to calculate the inhibition percentage of each treatment. Overall, all these compounds showed inhibition effects towards the growth of Oscillatoria sp., with the highest inhibition of $37 \%$ by $\mathrm{N}-((4-($ decyloxy)phenyl)carbamothioyl)-4-methyl benzamide at the concentration of 18 $\mu \mathrm{g} \cdot \mathrm{mL}^{-1}$. The methyl group that attach to the synthesized compound may contribute to the effectiveness of the compound which act as an algae inhibitor. However, extensive studies still need to be conducted in order to investigate the mechanism on how this compound reacts with Oscillatoria sp..
\end{abstract}

Key words: Eutrophication, alkoxyl thiourea, Oscillatoria sp., chlorophyll-a, inhibition effect.

\section{Introduction}

Nowadays, the aquatic environment has been greatly affected by various type of environmental problem. These environmental problem are closely related to the anthropogenic effects which mainly contributed by human activity. Water pollution becomes a major environmental problem that comes into concern to many of the community world widely. There are various sources of pollutants that contribute in increasing the concentration of the chemical contaminants in water resources such as industrials, constructions, agricultures and aquacultures [1]. The excess nutrient or contaminant from these activities may lead to the massive growing

Corresponding author: Nor Azman Kasan, Ph.D., research field: environmental science. E-mail: norazman@umt.edu.my. up of toxic blue green algae either in freshwater or seawater. One of the water pollution consequences is eutrophication problem. Eutrophication is commonly caused by the over enrichment of nutrients such as phosphate and nitrate which contribute to the depletion of oxygen in water $[2,3]$. This condition will then leading to the rapid growth of the toxic blue green algae or known as cyanobacteria which will caused Harmful Algal Blooms (HABs) phenomenon. HABs occurred when there is rapid increase of the growth of toxic blue green algae when there are excessive nutrients available in the water bodies. The increment of nutrients loading into water bodies causing the eutrophication will enhances the primary productivity which result in high rates of microbial 
activities [4] and finally, lead to human health problems. There are numbers of toxic blue-green algae species that potentially produce harmful toxins. As an example, Lyngbya robusta is one of the cyanobacteria species that can release hepatoxin and cyanotoxin once its blooming [5]. The blooming of toxic blue green algae producing cyanotoxin in certain concentration level can be very poisonous and lead to kill animals and humans community [6]. To overcome this problem, there are conventional approaches to treat HABs such as the usage of acidification, copper sulphate and barley straw methods [7-9]. As a novel method to treat HABs problem, many researches proved that the thiourea compound was successfully used as antimicrobial and antifungal agent [10-12]. Therefore, this study was aimed to investigate the potential usage of alkoxyl substituted thiourea derivatives namely $\mathrm{N}$-((4-(decyloxy)phenyl) carbamothioyl)-4-methyl benzamide (compound 1), $\mathrm{N}-((4-($ decyloxy)phenyl) carbamothioyl)-4-nitro benzamide (compound 2), N-((4-(decyloxy)phenyl) carbamothioyl)-4-chloro benzamide (compound 3), $\mathrm{N}-((4-($ decyloxy)phenyl) carbamothioyl) benzamide (compound 4) to inhibit the growth of toxic blue green algae, Oscillatoria sp. which was isolated from Kenyir lake, Terengganu, Malaysia and could be further used as anti-algae agent. However, due to the uniqueness and fair conjugation properties of thiourea derivatives of compounds 1 and 2 have been reported before in previous occasions within our group [13, 14].

\section{Material and Methods}

Toxic blue green algae, Oscillatoria sp. was successfully sampled at four selected sampling sites using a mesh size of $25 \mu \mathrm{m}$ phytoplankton net from Kenyir Lake, Terengganu, Malaysia (Fig. 1). In the laboratory, these toxic blue green algae species were isolated and identified based on their taxonomic identification key under light microscope [15, 16]. After a series of sub-culturing process, pure culture of Oscillatoria sp. was obtained. Using BG-11 agar

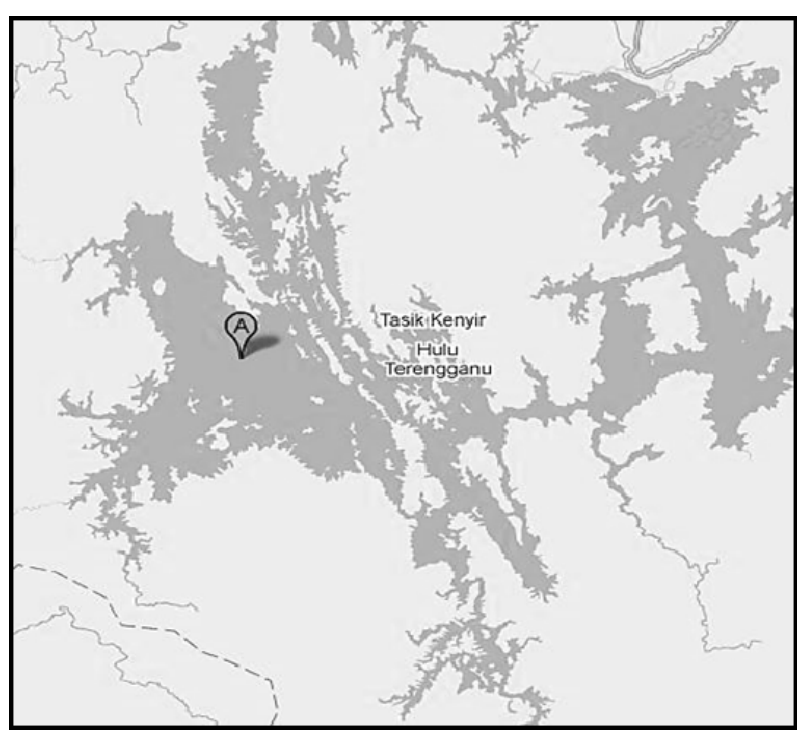

Fig. 1 Sampling location of toxic blue green algae at Kenyir lake, Terengganu, Malaysia.

media, this species was grown and maintained in pure culture. For inhibition treatment purposes, the pure culture of Oscillatoria sp. was transferred onto BG-11 liquid medium for mass culturing in $2 \mathrm{~L}$ conical flask. The mass culture of pure species was then harvested during exponential phase (6-8 days) for the inhibition effects treatment using four different alkoxyl thiourea derivatives compounds.

Four alkoxyl thiourea derivatives compounds were used throughout this study which was synthesized by Department of Chemical Sciences, School of Fundamental Science, Universiti Malaysia Terengganu (Table 1). All compounds (Table 1) were firstly diluted by adding $1,000 \mu \mathrm{g}$ of each compounds with $1 \mathrm{~mL}$ of dimethyl sulfoxide (DMSO) to obtain a total concentration of $1,000 \mu \mathrm{g} \cdot \mathrm{mL}^{-1}$. Two fold of serial dilution were carried out on five different concentrations resulted in final concentration of 16 $\mu \mathrm{g} \cdot \mathrm{mL}^{-1}, \quad 18 \mu \mathrm{g} \cdot \mathrm{mL}^{-1}, 20 \mu \mathrm{g} \cdot \mathrm{mL}^{-1}, 24 \mu \mathrm{g} \cdot \mathrm{mL}^{-1}$ and 28 $\mu \mathrm{g} \cdot \mathrm{mL}^{-1}$ respectively. Two milliliter of each compounds were added up into $30 \mathrm{~mL}$ of Oscillatoria sp. growth cultures in $100 \mathrm{~mL}$ conical flask. Controlled treatment was also set up by replacing the compound solution with distilled water. Finally, all the treatment cultures were incubated for 24 hours under continuous illumination at $25^{\circ} \mathrm{C}$. The inhibition 
Table 1 Molecular structure of four different types of alkoxyl thiourea derivatives.

No. $\quad$ Name

treatments were conducted during the exponential growth of Oscillatoria sp. (6-8 days, Fig. 3).

After 24 hours treatment periods, the control and treated Oscillatoria sp. cultures with four different types of alkoxyl thiourea derivatives were filtered through $0.45 \mu \mathrm{m}$ Whatman filter paper. The filtrates were washed off using $5 \mathrm{~mL}$ to $10 \mathrm{~mL}$ of $100 \%$ acetone in $50 \mathrm{~mL}$ centrifuge tubes for chlorophyll- $a$ (Chl-a) determination. The centrifuge tubes were then sealed with aluminum foil and left overnight at $4{ }^{\circ} \mathrm{C}$ in the dark. After 24 hours, the samples were then centrifuged for 10 minutes at $3,000 \mathrm{rpm}$. The supernatant was taken out and measured at 665 $\left(\mathrm{OD}_{665}\right), 645\left(\mathrm{OD}_{645}\right)$ and $630\left(\mathrm{OD}_{630}\right)$ using UV-Vis spectrophotometer. The chl- $a$ content for each centrifuge tubes was calculated using the formula as Eq. (1):

$$
\text { Chl- } a\left(\mu \mathrm{g} \cdot \mathrm{L}^{-1} \text { or } \mathrm{mg} \cdot \mathrm{m}^{-3}\right)=\frac{C a \times V a}{V c}
$$

Where,

$\mathrm{Ca}$ was $11.6 \times \mathrm{OD}_{665}-1.31 \times \mathrm{OD}_{645}-0.14 \times$ $\mathrm{OD}_{630} ; \mathrm{Va}$ was volume of acetone $(\mathrm{mL})$ used for extraction and $V c$ was volume of culture (L).

Furthermore, the inhibition effect in terms of percentage of compounds 1-4 to the growth of Oscillatoria sp. cultures were calculated using Eq. (2):

$$
\text { Inhibition effect }(\%)=\frac{C c-C t}{C c} \times 100
$$

Where,

Cc was Chl- $a$ concentration $\left(\mathrm{mg} \cdot \mathrm{m}^{-3}\right)$ of control (untreated), Ct was Chl- $a$ concentration $\left(\mathrm{mg} \cdot \mathrm{m}^{-3}\right)$ of treated culture.

\section{Results and Discussion}

Samples of Oscillatoria sp. were successfully isolated from Kenyir Lake, Terengganu, Malaysia. The fresh specimen of Oscillatoria sp. was observed under light microscope based on their specific morphological structures. The filamentous and cylindrical morphology of Oscillatoria sp. was determined (Fig. 2). The thallus of Oscillatoria sp. was cylindrical and filamentous, while the colour was bright to dull blue green. The filaments were branched falsely [17]. Besides, Oscillatoria sp. showed simple filament which consist of mucilaginous and plasma membrane sheath. The cell wall of Oscillatoria sp. lies between plasma membrane and mucilaginous sheath. The innermost layer was peptidoglycan layer. In most toxic blue green algae, the peptidoglycan varies between $1 \mathrm{~nm}$ and $10 \mathrm{~nm}$, but in Oscillatoria sp., it can reach up to $200 \mathrm{~nm}$ [17]. This species does not showed any heterocyst leading to be known as non-heterocystous blue green algae [18].

Growth curve of Oscillatoria sp. was plotted within 9 days (Fig. 3) showed that the maximum growth rate was up to 9 day's incubation periods. The lag phase of 


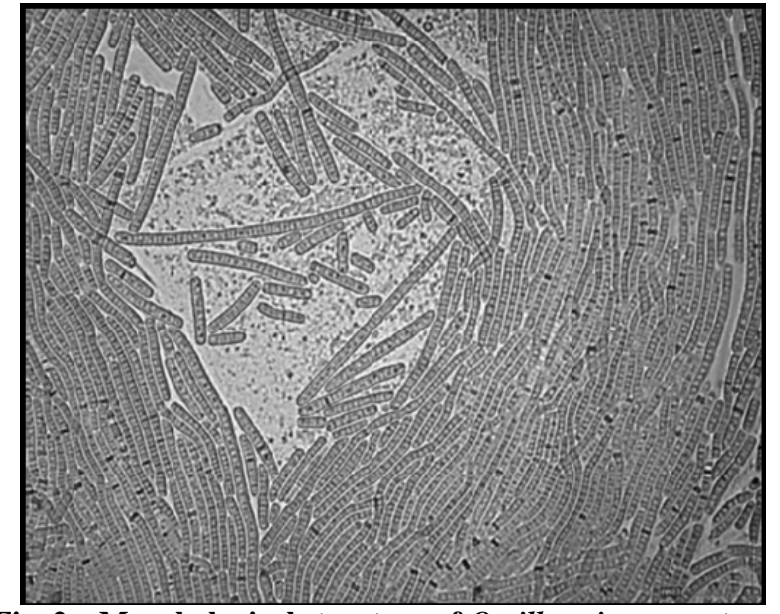

Fig. 2 Morphological structure of Oscillatoria sp. captured by microscope eyepiece camera, Dino-Eye under $40 \mathrm{X}$ magnification.

Oscillatoria sp. was between 1 days and 6 days in order for this species adapted to the new culture environment. The exponential phase was observed between 6 to 8 days, where the highest growth rate showed the most rapid growth. In comparison, Microcystis aeruginosa has longer exponential phase ranged between 6 days and 11 days [19]. It was important to determine the exponential phase of each isolated toxic blue green algae species for inhibitory treatment due to their maximum ability to consume nutrient sources for growth.

Four compounds of alkoxyl thiourea were used as candidates to be potential inhibitor towards the growth of toxic blue green algae, Oscillatoria sp. (Table 1). In general, it was proven that alkoxyl thiourea compounds were effectively acted as growth inhibitor for Oscillatoria sp. at the concentration of not less than $16 \mu \mathrm{g} \cdot \mathrm{mL}^{-1}$. Among all four compounds, the best candidate of alkoxyl thiourea compound that can inhibit the growth of Oscillatoria sp. was compound 1 at the concentration of $18 \mu \mathrm{g} \cdot \mathrm{mL}^{-1}$ with $37 \%$ of inhibition percentage (Fig. 4). However, at lower concentration $\left(16 \mu \mathrm{g} \cdot \mathrm{mL}^{-1}\right)$, compound 4 showed the highest inhibition percentage with up to $30 \%$ as comparing to the other compounds. In addition, similar amount of inhibition percentage was observed for compound 3 at the concentration of $20 \mu \mathrm{g} \cdot \mathrm{mL}^{-1}$. The treatment with compound 2 showed fluctuate trend where the highest inhibition percentage of $26 \%$ was observed at the concentration of $16 \mu \mathrm{g} \cdot \mathrm{mL}^{-1}$.

The leaving group that attached to alkoxyl substituted thiourea compounds showed different impacts to the inhibition percentage. It was observed that methyl group that attached to compound 1 showed the highest impact to inhibit the occurrence of HABs. As the results, this study showed different pattern of inhibition toward different concentration and different compounds. This might due to the effectiveness of inhibition percentage which definitely depends on the substituent that attach to the compounds. As an overall finding of this study, it was proven that all those compounds of alkoxyl subtituted thiourea derivatives showed the potential of inhibition effect towards the growth of Oscillatoria sp.. Thiourea

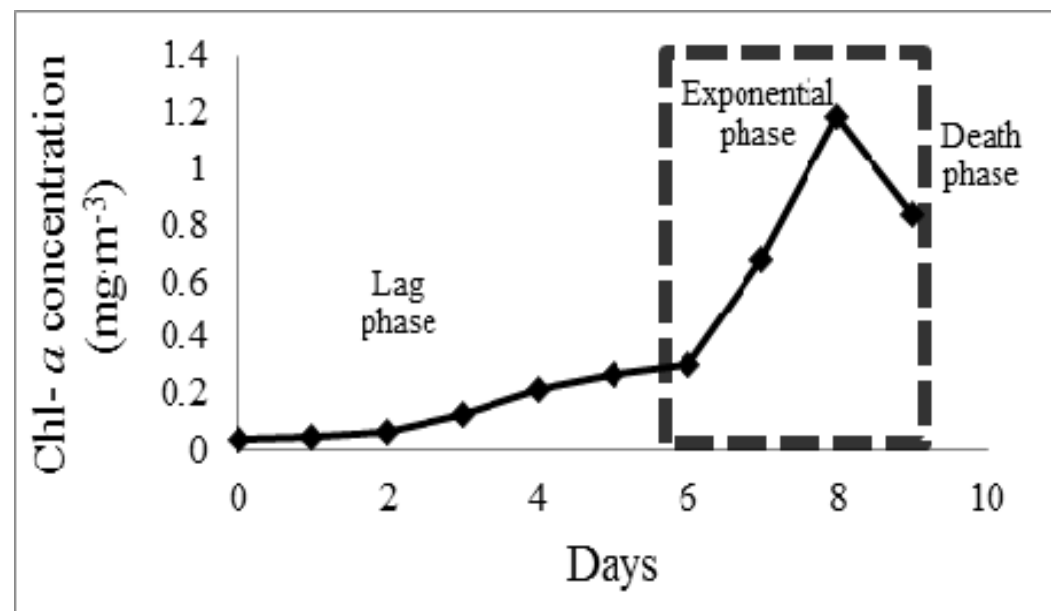

Fig. 3 Growth curve of Oscillatoria sp. determined by chl-a concentration from day 0 to day 9. 


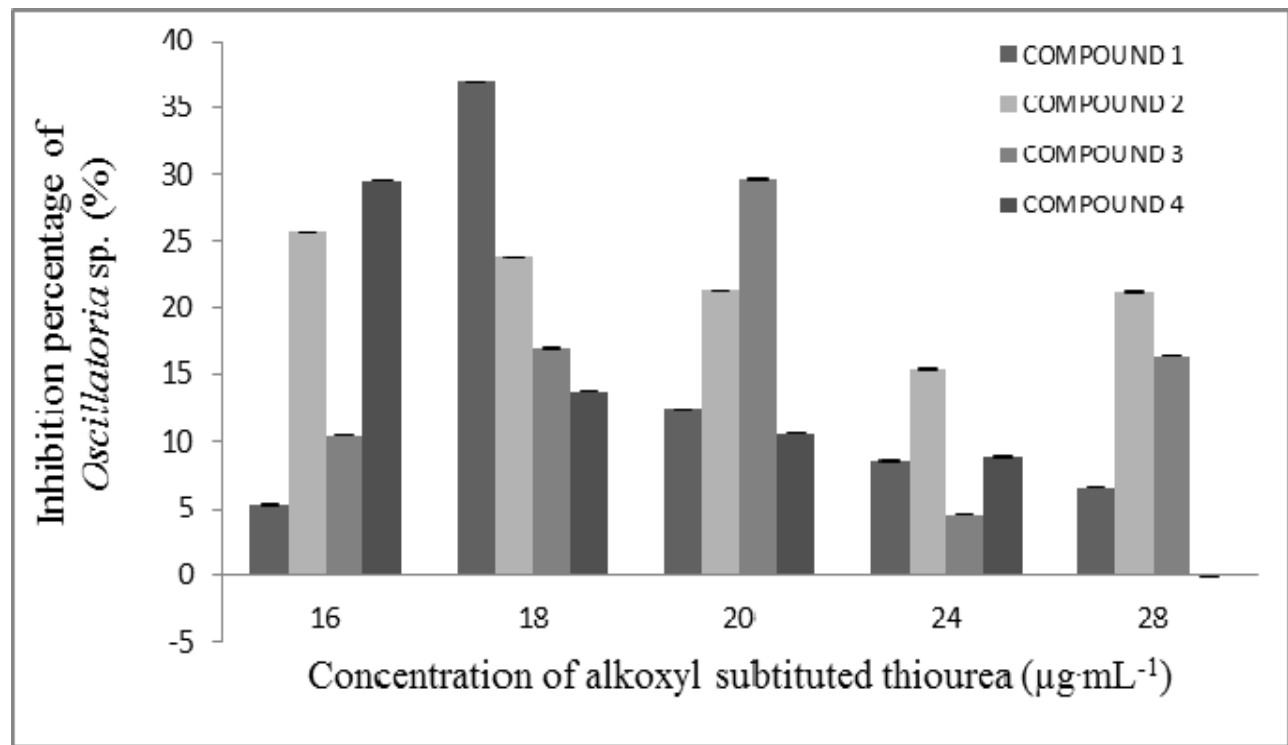

Fig. 4 Overall results showed the inhibition percentage of four different alkoxyl substituted thiourea compound, based on their chl-a concentration of control and treated cyanobacteria, Oscillatoria sp, data mean \pm standard deviation from three replicates.

derivatives of carboxymethyl chitosan showed inhibition effect toward the growth of three different types of bacteria species, Bacillis subtilis, Staphylococous aureus and Escherichia coli.. It was expected that inhibition of those bacteria were occurred through binding of chitosan with microbial DNA, which leads to the inhibition of the mRNA and protein synthesis via penetration of chitosan into the nuclei of the microorganisms. As an assumption toward the inhibition role of four different compounds of alkoxyl subtitued thiourea towards the growth of Oscillatoria sp., it possibly due to a significant changes in the cyanobacteria biomass, where the containing chl- $a$ in the cyanobacteria play an important role in the photosynthesis process. This assumption was made based on the reduction of chl- $a$ concentration for the determination of the inhibition percentage. The inhibition of alkoxyl substituted thiourea derivatives would distract the photosynthetic process. Consequently, the cyanobacteria will die due to lack of nutrients. On the other hand, the gamma radiation might give an adverse effect on the photosynthetic pigments. The level of carotenoids in other toxic blue green algae, Microcystis aeruginosa cells was reduced considerably in response to increasing doses of radiation [20].

\section{Conclusions}

Four compounds of alkoxyl thiourea derivatives were successfully showed an inhibition effects toward the growth of toxic blue green algae, Oscillatoria sp.. With the highest inhibition percentage at low amount of concentration, it can be concluded that N-((4-(decyloxy)phenyl)carbamothioyl)-4-methyl benzamide (compound 1) can be used as potential candidate for toxic blue green algae growth inhibitor. Even though the inhibition percentage was considerably low with less than $50 \%$, there was still sign of inhibition that need to be investigated. In conclusion, alkoxyl thiourea derivatives have high potential to be developed as the algaecidal agent but further study on this system should be conducted towards their mechanisms of inhibition.

\section{Acknowledgements}

The authors would like to thank the Ministry of Education, Malaysia for financial support under Exploratory Research Grant Scheme, ERGS (Vot no. 55096). Also, to all postgraduate students and all lab staffs at the School of Fisheries and Aquaculture 
Sciences and Aquaculture Tropical Institute (AKUATROP), UMT for their man power supports and cooperation throughout the study periods.

\section{References}

[1] Tortora, G. J., Funke, B. R., and Case, C. L. 2010. Microbiology an Introduction, 10th Ed.. San Francisco: CA Pearson Benjamin Cumming.

[2] Huang, C., Wang, X., Yang, H., Li, Y., Wang, Y., and Chena, X. et al. 2014. "Satellite Data Regarding the Eutrophication Response to Human Activities in the Plateau Lake Dianchi in China from 1974 to 2009." Science of the Total Environment 485-486: 1-11.

[3] Skogen, M. D., Eilola, K., Hansen, J. L. S., Markus, Meier, H. E., Molchanov, S. M., and Ryabchenko, V. A. 2014. "Eutrophication Status of the North Sea, Skagerrak, Kattegat and the Baltic Sea in Present and Future Climates: A Model Study.” Journal of Marine Systems 132: 174-184.

[4] Reddy, K. R., and Delaune, R. D. 2008. Biogeochemistry of Wetlands Science and Applications. America: Taylor and Francis Group, CRC Press.

[5] Rejmankova, E., Komarek, J., Dix, M., Komarkova, J., and Girone, N. 2011. "Cyanobacterial Blooms in Lake Atitlan, Guatemala.” Limnologica 41: 296-302.

[6] Huynh, L., Huwyler, J., Micheal, W., and Manfred, K. 2006. "Computational Aqueous Solubility Prediction for Drug Like Compounds in Congeneric Series." European Journal of Medicinal Chemistry 43: 501-512.

[7] Jia, Y., Wang, Q., Chen, Z., Jiang, W., Zhang, P., and Tian, X. 2010. "Inhibition of Phytoplankton Species by Co-culture with a Fungus." Ecological Engineering 36: 389-1391.

[8] Parker, D. L., Kumar, H. D., Rai, L. C., and Singh, J. B. 1997. "Potassium Salts Inhibit Growth of the Cyanobacteria Microcystis Spp. in Pond Water and Defined Media: Implications for Control of Microcystin-Producing Aquatic Blooms." Applied and Environmental Microbiology 63: 2324-2329.

[9] Wang, X., Hao, C., Zhang, F., Feng, C., and Yang, Y. 2011. "Inhibition of the Growth of Two Blue-Green Algae Species (Microsystis Aeruginosa and Anabaena Spiroides) by Acidification Treatments Using Carbon Dioxide." Bioresource Technology 102: 5742-5748.

[10] Faiz, A. R. 2013. "Inhibition of Alkoxyl Substituted Thiourea to the Growth of cyanobacteria (Oscillatoria sp.)." Master thesis, University Malaysia Terengganu.

[11] Mohamed, N. A., and Abd El-Ghany, N. A. 2012. "Preparation and Antimicrobial Activity of Some Carboxymethyl Chitosan Acylthiourea Derivatives." International Journal Biological Macromolecules 50: 1280-1285.

[12] Saeed, S., Rashid, N., Jones, P. G., Ali, M., and Hussain, R. 2010. "Synthesis, Characterization and Biological Evaluation of Some Thiourea Derivatives Bearing Benzothiazole Moiety as Potential Antimicrobial and Anticancer Agents." European Journal of Medicinal Chemistry 45: 1323-1331.

[13] Khairul, W. M., Mokthar, K. A., Isa, M. I. N., Samsudin, A. S., Adli, H. K., and Ghazali, S. R. et al. 2014. "Synthesis and Characterization of Nitrobenzoylthiourea Derivatives as Potential Conductive Biodegradable Thin Films." Phosphorus, Sulfur and Silicon and the Related Elements 189: 640-651.

[14] Saidatul Radhiah Ghazali, K. Ku Bulat, Isa, M. I. N., Samsudin, A. S., and Wan Khairul, M. 2014. "Contribution of Methyl Substituents on the Conductivity Properties and Behaviour of CMC-Alkoxy Thiourea Polymer Electrolyte." Molecular Crystal and Liquid Crystal 604: 126-141.

[15] Komarek, J., Komarkova, J., and Kling, H. 2003. "Filamentous Cyanobacteria." Freshwater Algae of North America 4: 117-196.

[16] Yang, S., Dong, L., Jiang, C., Cheng, X., Zhang, H., and Cui, L. 2007. "Isolation, Culture and Identification of Microcystis in Source Water." Life Science Journal 4: 28-32.

[17] Barsanti, L., and Gualtieri, P. 2006. Algae: Anatomy, Biochemistry and Biotechnology. America: Taylor and Francis Group, CRC Press.

[18] Bergman, B., Gallon, J. R., Rai, A. N., and Stal, L. J. 1997. " $\mathrm{N}_{2}$ Fixation by Non-heterocystous Cyanobacteria." FEMS Microbiology Reviews 19: 139-185.

[19] Backer, L. C. 2002. "Cyanobacterial Harmful Algal Bloom (Cyanohabs): Developing a Public Health Response." Lake and Reservoir Management 18 (1): 20-31.

[20] Zheng, B., Zheng, Z., Zhang, J., Luo, X., Liu, Q., and Wang, J. et al. 2012. "The Removal of Microcystis Aeruginosa in Water by Gamma-Ray Irradiation." Separation and Purification Technology 85: 165-170. 